\title{
Alu elements mediate large SPG11 gene rearrangements: further spatacsin mutations
}

\author{
Maria Conceição Pereira, MSc ${ }^{1,2}$, José Leal Loureiro, MD ${ }^{1,3}$, Jorge Pinto-Basto, MD ${ }^{1,2,4}$, \\ Eva Brandão, MD ${ }^{1,3}$, Ana Margarida Lopes, BSc², Georgina Neves, MD, Pureza Dias, MD ${ }^{6}$, \\ Ruth Geraldes, MD7, Isabel Pavão Martins, MD, PhD77, Vitor Tedim Cruz, MD³, \\ Erik-Jan Kamsteeg, MD, $\mathrm{PhD}^{8}$, Han G. Brunner, MD, $\mathrm{PhD}^{8}$, Paula Coutinho, MD, $\mathrm{PhD}^{1,3}$, \\ Jorge Sequeiros, MD, $\mathrm{PhD}^{1,2,4}$ and Isabel Alonso, $\mathrm{PhD}^{1,2,4}$
}

Purpose: Hereditary spastic paraplegias compose a group of neurodegenerative disorders with a large clinical and genetic heterogeneity. Among the autosomal recessive forms, spastic paraplegia type 11 is the most common.

Methods: To better understand the spastic paraplegia type 11 mutation spectrum, we studied a group of 54 patients with hereditary spastic paraplegia. Mutation screening was performed by PCR amplification of SPG11 coding regions and intron boundaries, followed by sequencing. For the detection of large gene rearrangements, we performed multiplex ligation-dependent probe amplification.

Results: We report 13 families with spastic paraplegia type 11 carrying either novel or previously identified mutations. We describe a complex entire SPG11 rearrangement and show that large gene rearrangements are frequent among patients with spastic paraplegia type 11 . Moreover, we mapped the deletion breakpoints of three different large SPG11 deletions and provide evidence for Alu microhomology-mediated exon deletion.

Conclusion: Our analysis shows that the high number of repeated elements in SPG11 together with the presence of recombination hotspots and the high intrinsic instability of the $15 \mathrm{q}$ locus all contribute toward making this genomic region more prone to large gene rearrangements. These findings enlarge the amount of data relating repeated elements with neurodegenerative disorders and highlight their importance in human disease and genome evolution.

Genet Med 2012:14(1):143-151

Key Words: Alu-mediated nonallelic homologous recombination; hereditary spastic paraplegia; large gene rearrangements, spatacsin; SPG11

(SPG11 and SPG15) suggest an involvement of these three proteins in the same pathway.

Among HSP genes, SPG11 has a high mutation rate, and notwithstanding its size, it is actually the most frequently mutated gene in autosomal recessive spastic paraplegia with thin corpus callosum. To date, more than 100 different mutations ${ }^{1-5,9-23}$ have been reported worldwide, in a total of 128 families with complex autosomal recessive spastic paraplegia.

In this study, we report 13 new families with SPG11 presenting novel or previously described mutations, as well as the molecular mechanism responsible for large deletions in this gene; we also discuss the high frequency of the large gene rearrangements identified in this gene.

in the central nervous system; recent immunocytochemistry assays showed that spatacsin is distributed throughout the cytoplasm and is probably involved in axonal transport. ${ }^{6} \mathrm{~A}$ possible involvement in DNA repair has also been suggested; in fact, a new HSP disease-causing gene was recently identified (KIAA0415, responsible for SPG48) and its product was found to be involved in DNA repair. ${ }^{7}$ Moreover, this protein is an interactor of two other HSP proteins, spatacsin and spastizin, whose overlapping brain expression patterns ${ }^{8}$ and clinical traits

\section{MATERIALS AND METHODS}

\section{Patients}

We selected a group of 54 unrelated Portuguese patients with HSP to screen for SPG11 mutations: 19 cases with a suggestive pattern of autosomal recessive inheritance and 35 isolated cases. Twenty-one patients presented with pure HSP, whereas 33 had a complex phenotype, with mental impairment, neuropathy,

\footnotetext{
${ }^{1}$ UnIGENe, IBMC, Porto, Portugal; ${ }^{2}$ CGPP, IBMC, Porto, Portugal; ${ }^{3}$ Serviço Neurologia, Hospital São Sebastião, Feira, Portugal; ${ }^{4}$ ICBAS, University of Porto, Porto, Portugal; ${ }^{5}$ Serviço Neurologia, Centro Hospitalar de Vila Real/Peso da Régua, Vila Real, Portugal; ${ }^{6}$ Serviço Neurologia, Hospital Santo André de Leiria, Leiria, Portugal; ${ }^{7}$ Serviço Neurologia, Hospital de Santa Maria, Lisboa, Portugal; ${ }^{8}$ Department of Human Genetics, Radboud University Nijmegen Medical Centre, Nijmegen, The Netherlands. Correspondence: Isabel Alonso (ialonso@ibmc.up.pt)
} 

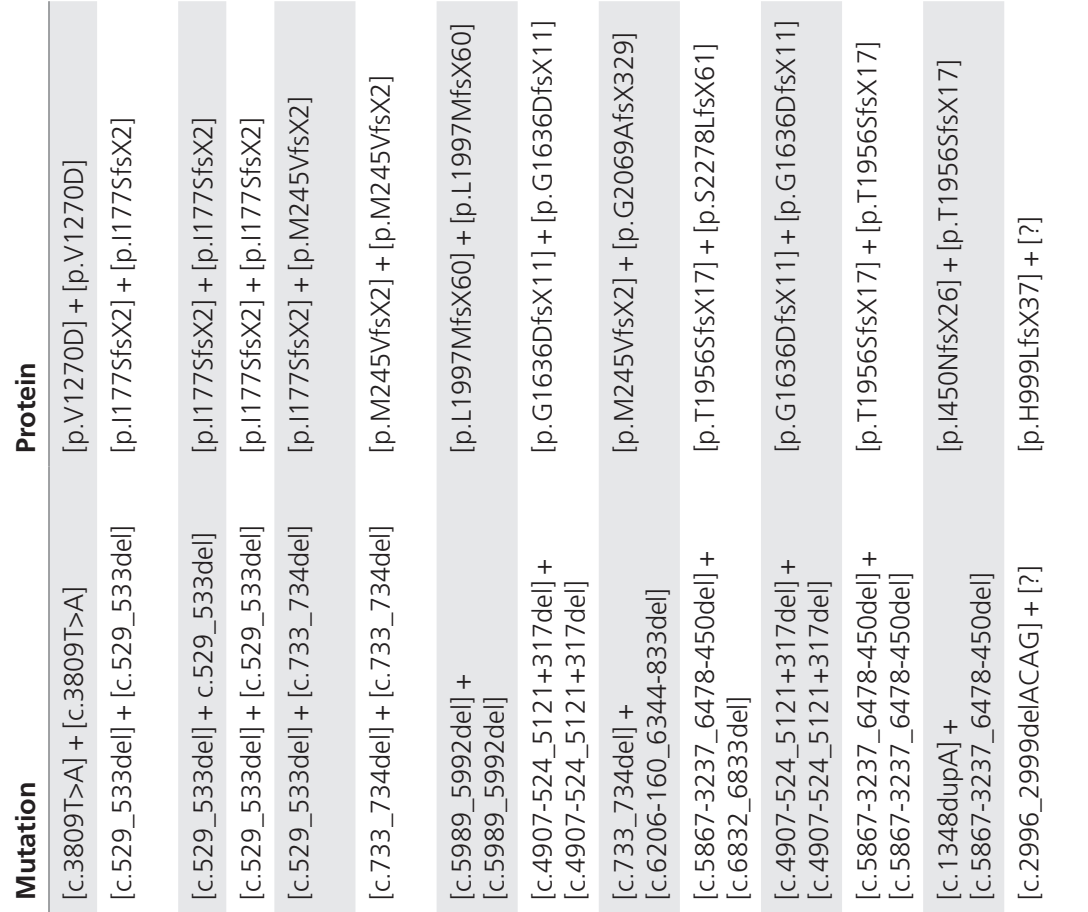

๖ํํำเ

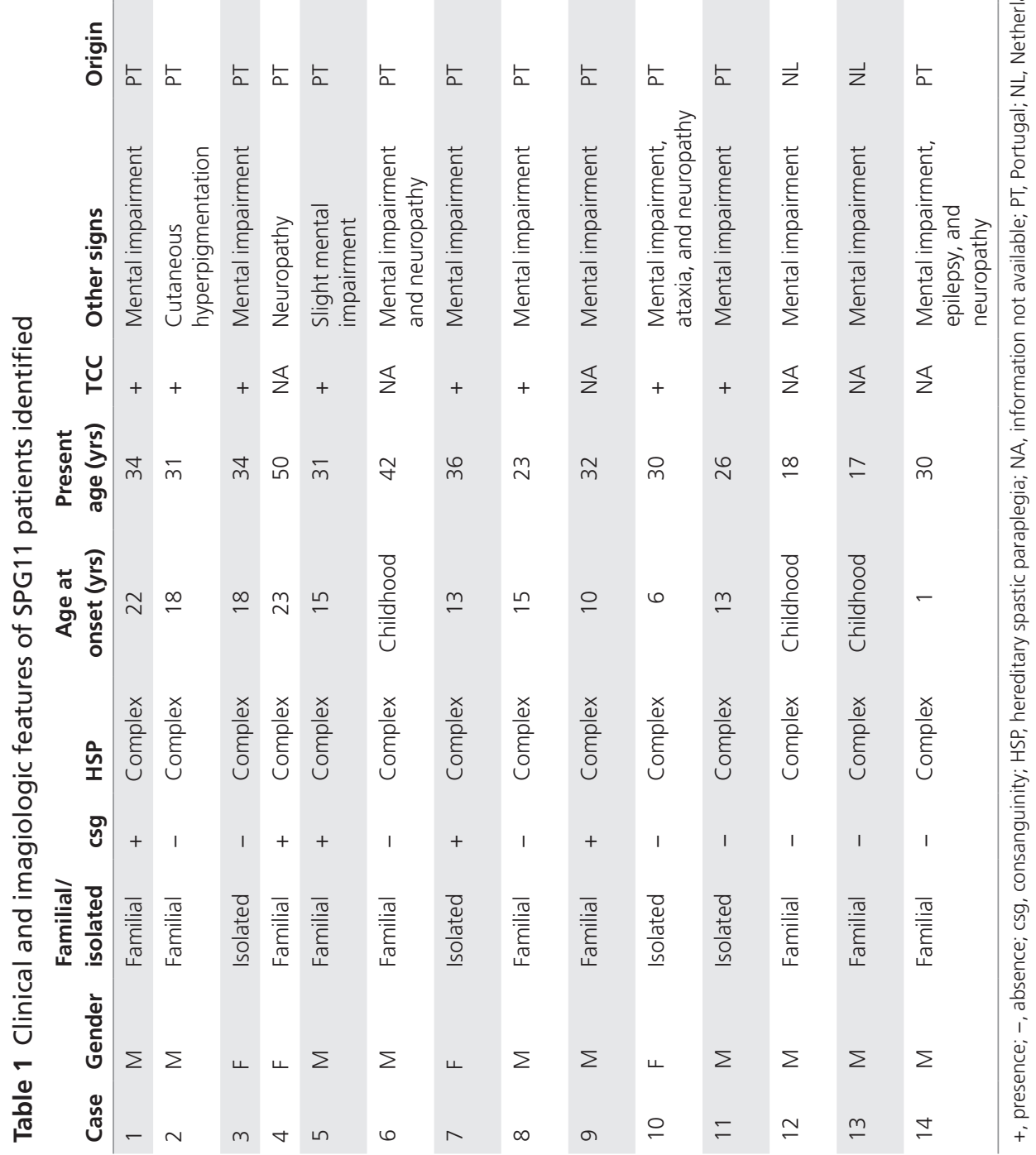

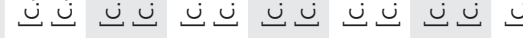


and/or epilepsy in addition to the spasticity of the lower limbs. Some patients presented with a thin corpus callosum, white matter hyperintensity, or medullar atrophy on brain MRI. In addition, SPG11 mutation screening was also performed in two Dutch families.

\section{Mutation screening}

Peripheral blood was collected from patients and their relatives after written informed consent was obtained. Genomic DNA was extracted from peripheral blood leukocytes through salting out, ${ }^{24}$ and specific primers were designed for all coding regions (including splice site boundaries and intronic flanking regions) of the SPG11 gene (RefSeq NM_025137.3), using PrimerQuest. Each exon was amplified by PCR with HotStarTaq Master Mix (Qiagen, Hilden, Germany), directly sequenced with the Big Dye Terminator Kit v1.1 (Applied Biosystems, Carlsbad, CA), and loaded on an ABI 3130xl Genetic Analyzer (Applied Biosystems, Carlsbad, CA).

\section{Multiplex ligation-dependent probe amplification}

To detect large gene rearrangements (deletions or duplications), multiplex ligation-dependent probe amplification (MLPA) was performed using the Salsa MLPA kit P306-A1 (MRC-Holland, Amsterdam, The Netherlands), according to the manufacturer's instructions. Fragments were analyzed on an ABI 3130xl Genetic Analyzer using 250-LIZ (Applied Biosystems, Carlsbad, CA), as a size standard, and GeneMarker v1.90 (SoftGenetics, State College, PA).

\section{Long-range PCR and breakpoint analysis}

Long-rangePCR was performed on genomic DNA from probands to confirm the large SPG11 deletions detected by MLPA; breakpoints were determined by direct sequencing. The reaction used the Expand Long template PCR system (Roche Diagnostics, Manheim, Germany), according to the manufacturer's instructions. Primers for the exons upstream and downstream of the deletion were used and the deletion breakpoints were afterwards narrowed down by primer walking. Finally, deletion junctions were amplified with specific primer sequences, followed by direct sequencing for breakpoint confirmation.

\section{In silico analysis}

All new mutations found were analyzed with Alamut v2.0 (Interactive Biosoftware, Rouen, France). The entire SPG11 gene (NCBI36:15:42641587:42743768:-1), including 600 bp upstream and downstream of the $5^{\prime}$ and $3^{\prime}$ untranslated regions, was analyzed using both RepeatMasker (http://www. repeatmasker.org) and CENSOR (http://www.ebi.ac.uk/Tools/ censor) to identify interspersed repeats. Sequence identities of Alu repeat signatures of interest were evaluated with the National Center for Biotechnology Information (NCBI) BLASTN tool; the homologous genes of four primate species (Gorilla gorilla, Pan troglodytes, Pongo pygmaeus, and Macaca mulatta) were also analyzed with RepeatMasker and NCBI BLASTN for comparison with the human gene regarding the presence or absence of the particular Alu elements of interest. Finally, COMPASS ${ }^{25}$ was used to search for complex motifs, degenerated from the sequence CCNCCNTNNCCNC, in the human SPG11 gene.

\section{RESULTS}

\section{SPG11 mutations in patients with spastic paraplegia}

Mutation screening and gene dosage analysis of SPG11 allowed the identification of disease-causing mutations in 14 of 54 unrelated HSP patients tested. Through SPG11 direct sequencing, we identified 6 point mutations (mostly small deletions) including one novel missense and a 4-bp deletion. MLPA allowed us to detect three new large gene rearrangements-two different single-exon deletions, as well as multiple exon deletions, and an interesting case of a heterozygous complex gene rearrangement. The main clinical features and the mutations of the diagnosed patients are summarized in Table 1; the presence of the identified mutations was confirmed in additional affected family members as well as its parental segregation.

The first patient studied (case 1) carried a novel homozygous 1-bp exchange, c.3809T $>\mathrm{A}$ in exon 22, resulting in a missense substitution of a valine at protein position 1270 for an aspartic acid (Figure 1a), a very conserved residue. Of interest, patients 8 and 11 were found to be homozygous for a novel large deletion encompassing exon 29. PCR of this exon did not result in fragment amplification, and MLPA confirmed the homozygous deletion. Although they share the same mutation, the two patients originate from different geographical regions. Patient 9 was found to be a compound heterozygous carrier of the c.733_734delAT, in exon 4, and a novel large deletion comprising exon 33 detected by MLPA. Patient 10 presented a more challenging case as she was found to be compound heterozygous for the c.6832_6833delAG deletion in exon 37 and a complex entire gene rearrangement comprising the duplication of exons 1 to 30 and 35 to 40 in combination with a deletion that encompasses exons 31 to 34 (Figure 1b). The duplicated area extended at least to the flanking genes CASC4 and B2M, also detected by the MLPA probe mix. This four-exon deletion was also present in two Dutch families, although not associated with this complex rearrangement. Last, we identified a novel frameshift mutation in patient 14, c.2996_2999delACAG, in exon 16, changing a histidine to a leucine at position 999, leading to an altered reading frame and a premature stop codon after 36 residues (p.His999LeufsX37) (Figure 1c). This patient had a complex form of spastic paraplegia, with neuropathy, epilepsy, and mental impairment, and a family history of the disease; however, after mutation screening and MLPA, we could find only this small deletion in the heterozygous state, thereby being unable to establish a diagnosis of SPG11.

Breakpoint deletion determination and deletion mechanism The breakpoints of the 1056-bp deletion (exon 29) were mapped by primer walking to $524 \mathrm{bp}$ upstream and $317 \mathrm{bp}$ downstream of exon 29 (c.4907-524_5121+317del) in both patients. This deletion (Figure 2a) leads to a frameshift, generating a premature 

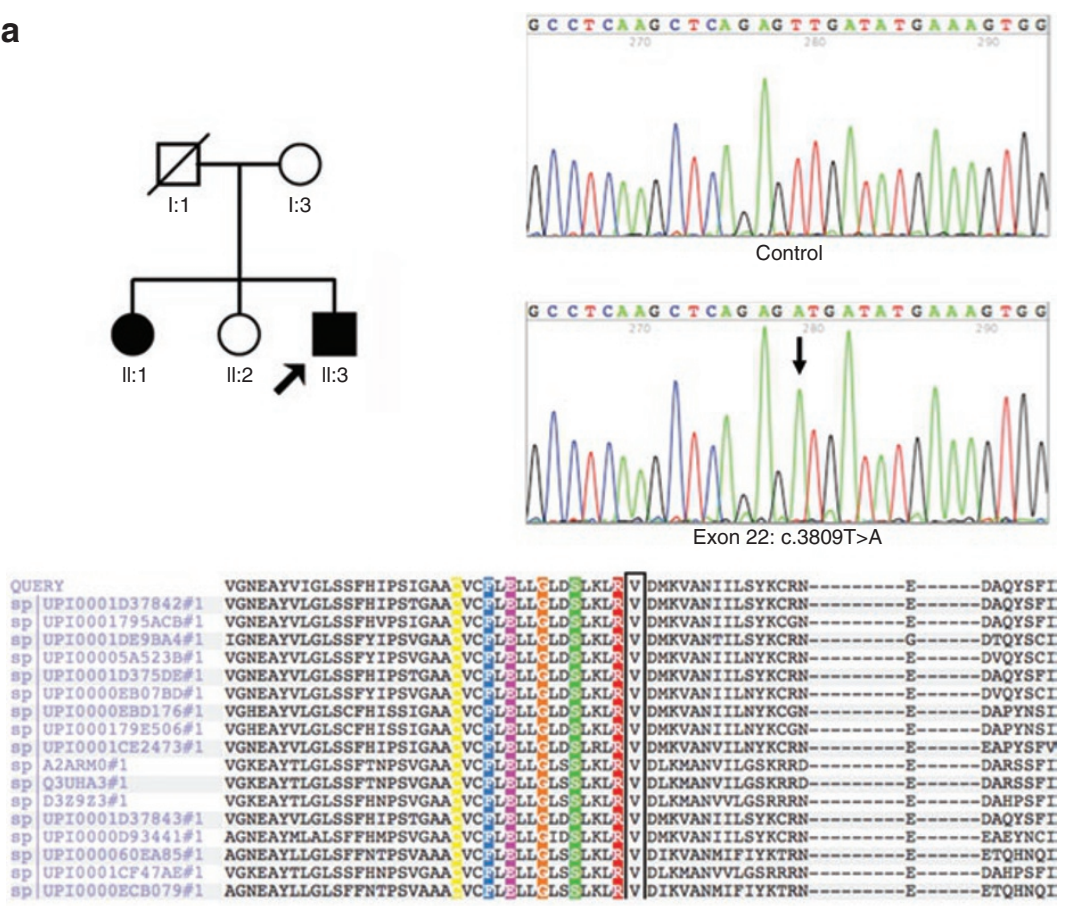

b
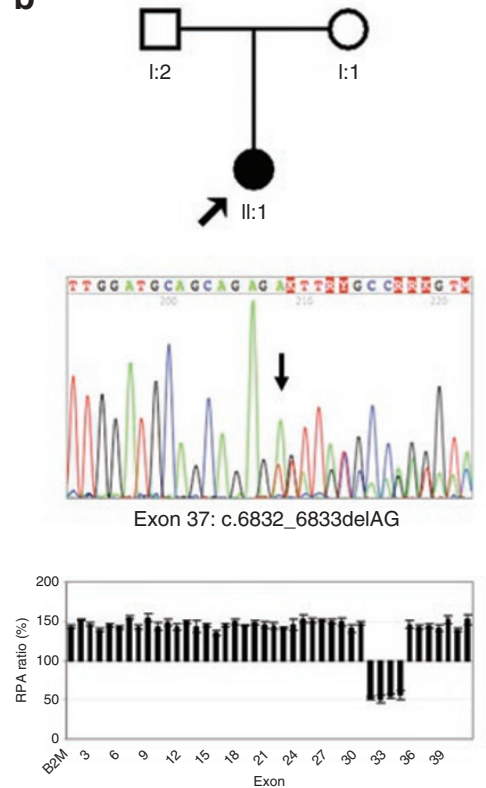
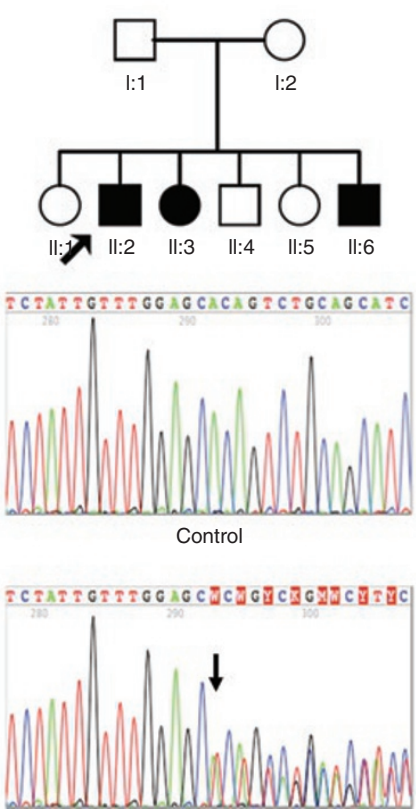

Exon 16: c.2996_2999delACAG

Figure 1 Pedigrees of three families of our cohort. (a) Family pedigree of proband 1, in whom we found a novel homozygous missense mutation (p.V1270D) (black arrow in the electropherogram, upper panel). Multiple sequence alignment of spatacsin with other species shows the conservation of this amino acid as well as the adjacent sequence. (b) Family of proband 10 (upper panel); electropherogram showing a heterozygous 2-bp deletion in exon 37 and a complex heterozygous gene rearrangement identified by multiplex ligation-dependent probe amplification, involving duplications and a deletion (lower panel). (c) Family of proband 14 (upper panel); electropherogram showing a heterozygous 4-bp deletion in exon 16 (lower panel). RPA, relative peak area.

stop codon (p.Gly1636AspfsX11). Through bioinformatic analysis, we were able to colocalize both $5^{\prime}$ and $3^{\prime}$ breakpoints within Alu elements, namely an AluSz in intron 28 and an AluY localized in intron 29 . These elements are characterized by a high degree of homology ( $77 \%$ identity); moreover, there is a 24-bp region of microhomology adjacent to each breakpoint.
Regarding the exon 33 deletion (Figure 2b), its breakpoints were mapped to 169 bp upstream of exon 33 and 833 bp upstream of exon 34 (c.6206-160_6344-833del), resulting in a loss of $1489 \mathrm{bp}$. This large rearrangement also produces a frameshift, giving rise to a premature stop codon (p.Gly2069AlafsX329). Similar to the previous rearrangement 

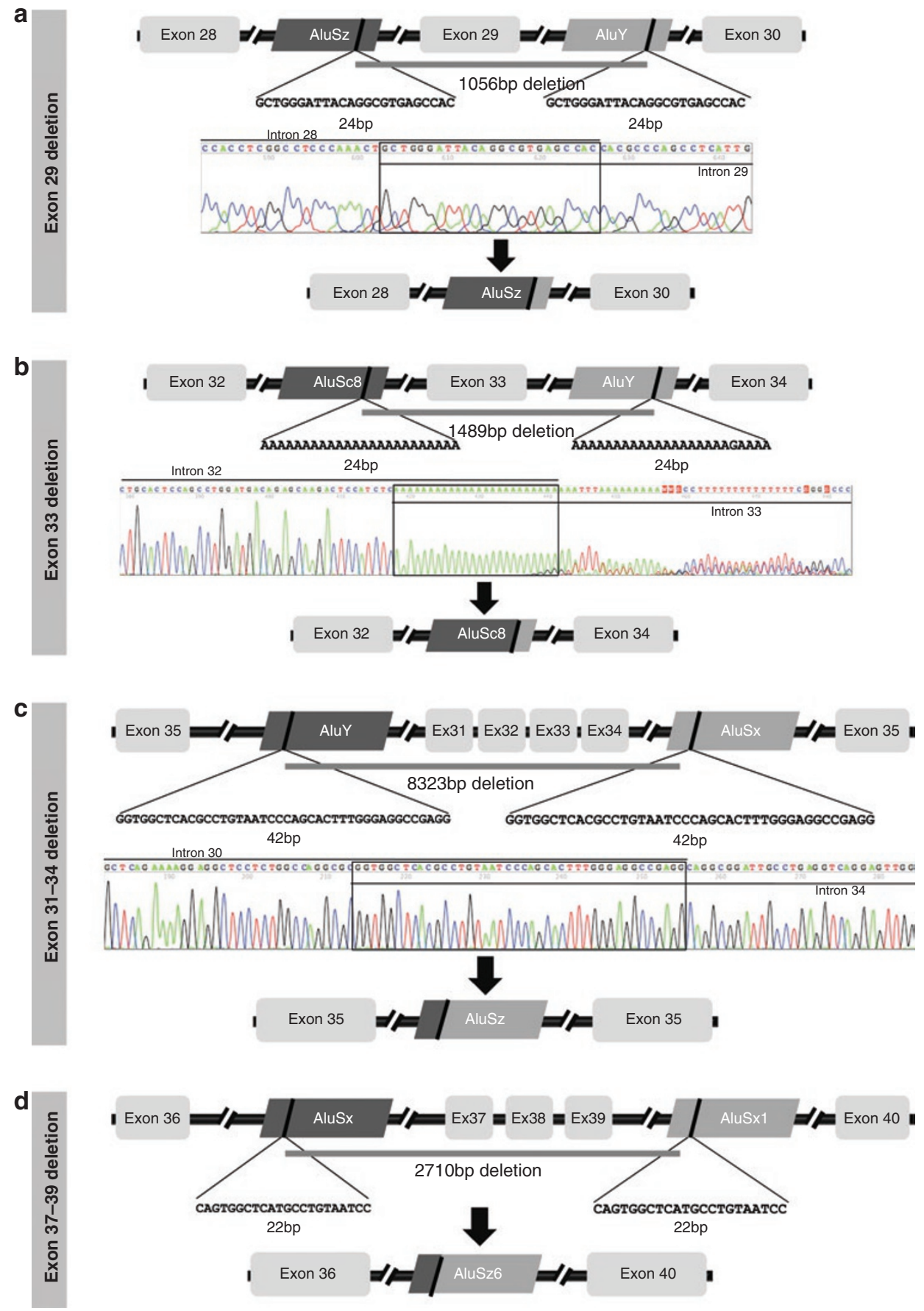

Figure 2 Characterization of the spastic paraplegia type 11 (SPG11) intragenic deletions found in this study and schematic representation of its breakpoints. (a) Breakpoint deletion for exon 29 found in two Portuguese families; (b) representation of the exon 33 deletion found in one Portuguese family (showing contamination with wild-type allele in the sequencing product; nevertheless, sequence deconvolution confirms the deletion breakpoints); (c) exon 31 to 34 deletion breakpoint from two Dutch and one Portuguese patient; (d) breakpoint analysis of a previously reported SPG11 deletion shows the same rearrangement mechanism. An Alu-mediated mechanism appears to be responsible for all these deletions.

described, both $5^{\prime}$ and $3^{\prime}$ breakpoints also coincide with Alu elements (with the $5^{\prime}$ breakpoint within an AluSc8 in intron 32, and the 3' breakpoint within an AluY in intron 33), with high degree of homology ( $86 \%$ identity) between them. Furthermore, this breakpoint matches a 19-bp microhomology domain.
The breakpoints for the four-exon deletion (Figure 2c) were also mapped (c.5867-3237_6478-450del) in the Portuguese and both Dutch families; likewise, it also results in a frameshift and a premature stop codon after 16 residues (p.T1956SfsX17). The boundaries of this 8323-bp deletion, again, overlap Alu elements (5' within an AluY 


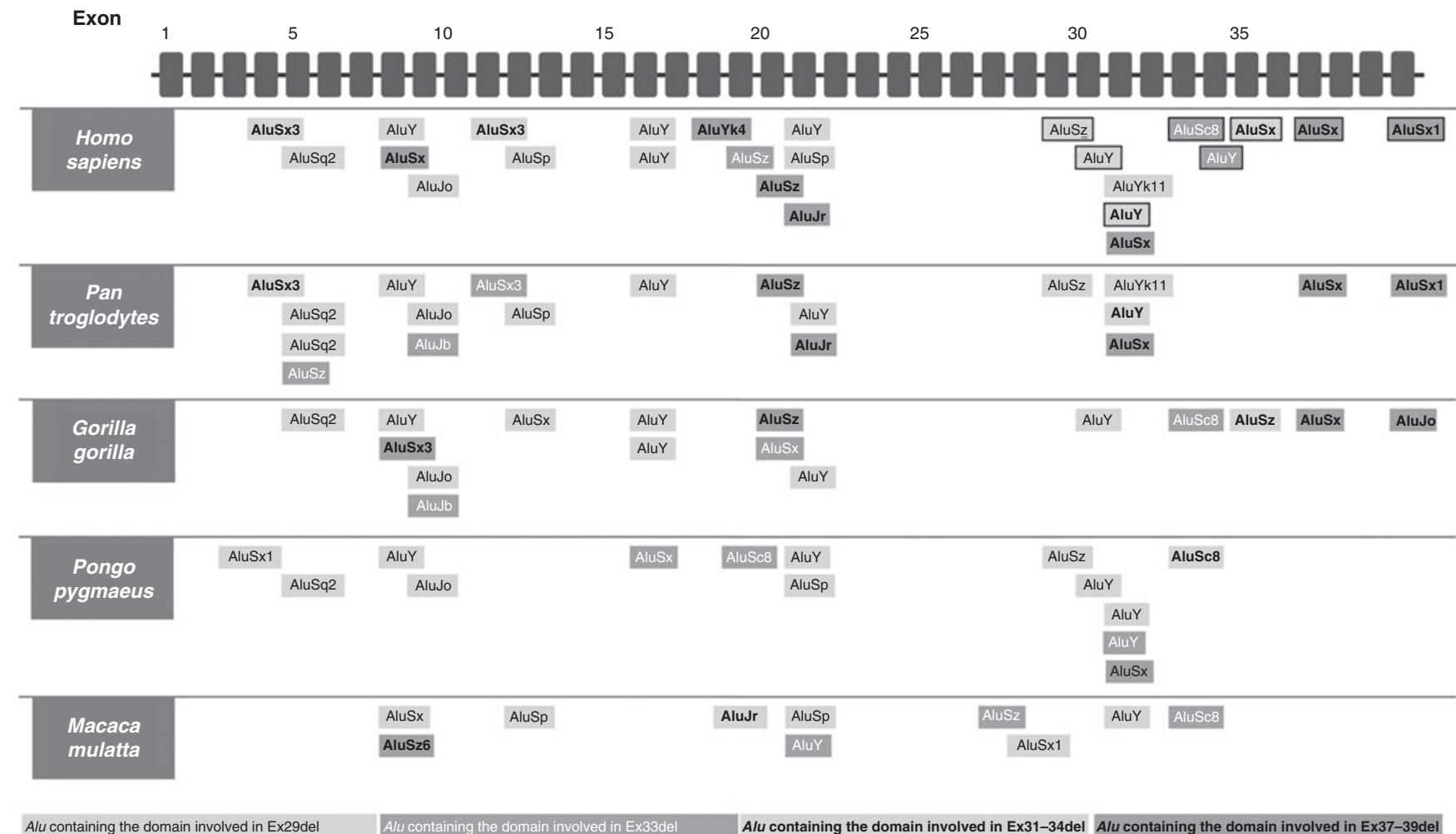

Figure 3 Evolutionary conservation of the Alu sequences involved in spastic paraplegia type 11 (SPG11) deletions in nonhuman primates. The Alu elements found in this study to be involved in SPG11 deletions are boxed. Additional regions within the SPG11 gene showing these microhomology domains, as well as the evolutionary conservation of the different Alus in nonhuman primates, are also presented.

in intron 30 and the 3' coinciding with an AluSx in intron $34)$, possessing an adjacent 42 -bp region of microhomology and a high degree of identity (79\%). All results found with RepeatMasker for interspersed repeats were confirmed with the CENSOR software; a total of 189 masked elements were found, representing $55 \%$ of the SPG11 gene. Also, COMPASS allowed the identification of 13 degenerate sequences from the CCNCCNTNNCCNC motif, distributed throughout the gene, and totaling 23 occurrences.

\section{DISCUSSION}

\section{Spatacsin mutation frequency in patients with spastic paraplegia}

The SPG11 mutational spectrum of our cohort comprises mostly small deletions, together with three large-scale rearrangements (six different families), all distributed throughout the SPG11 gene.

Of interest, we found two recurrent mutations, c.529_533delATATT and c.733_734delAT, which together account for $41 \%$ of the mutated alleles. A founder effect has been suggested for a few mutations in SPG11 patients from the Mediterranean basin. ${ }^{5}$ In particular, a haplotype analysis has shown that both Portuguese and Brazilian patients with the c.529_533delATATT mutation share the same haplotype, pointing toward a common mutational origin. ${ }^{4}$ The 2-bp deletion in exon 4 (c.733_734delAT) is one of the two most frequent mutations worldwide ${ }^{1,3-5,11-13,16,22}$, which might suggest either the presence of a mutational hotspot or a significantly old mutational event. Another interesting finding in this study is the frequency of large deletions, which appear in almost half of our index cases.

In addition, we have also found a novel missense mutation (V1270D) that replaces a highly conserved hydrophobic residue by a negatively charged hydrophilic amino acid (Figure 1a). All Web-based variant scoring algorithms used by Alamut (PolyPhen-2, SIFT, Align GVGD), as well as PANTHER, ${ }^{26}$ SNAP, ${ }^{27}$ PMut, ${ }^{28}$ and $\mathrm{PhD}-\mathrm{SNP},{ }^{29}$ predicted this mutation to be pathogenic. This substitution is located adjacent to the third highly conserved $\alpha$-helix ${ }^{3}$ and thus could have a major impact on protein folding.

The novel 4-bp deletion results in a premature termination codon in exon 16, and although we could not confirm SPG11 diagnosis in the patient carrying this deletion, we cannot fully exclude it either, since the second disease-causing mutation may be located in regions that our genetic screen does not cover: the promoter, the $3^{\prime}$ or $5^{\prime}$ untranslated regions (intronic flanking regions required for splicing were covered by our analysis). As a general rule, only premature termination codons (PTCs) located <50-55 nucleotides upstream, or downstream of the last exon-exon junction, manage to escape nonsense-mediated decay (NMD). ${ }^{30}$ Hence, the mutant transcript in question is probably targeted for degradation, because it is located closer to the $5^{\prime}$ end of the mRNA and upstream of several exon-exon 
junctions. Nevertheless, there are exceptions to this rule. Namely, it was recently shown that a PTC-causing mutation in the middle of the $\mathrm{GABA}_{\mathrm{A}}$ receptor GABRA1 escapes $\mathrm{NMD} ;{ }^{31}$ thus, we can neither confirm nor exclude SPG11 diagnosis.

In contrast, the large deletion found in patient 9, which causes exon 33 skipping, leads to a frameshift and a premature termination codon after 328 amino acids, meaning that, if translated, the protein would have almost a full-length size (2,397 amino acids), i.e., only 46 amino acids shorter than the wild type. Assuming a correct splicing of the remaining exons downstream of the deleted portion, and considering that the PTC is positioned after the last exon-exon junction, it is highly probable that this transcript escapes NMD, originating an almost full-length protein with a mutant C-terminal. This novel spatacsin C-terminus may behave differently from the normal protein, by abnormal protein interaction or trafficking, which could result in a more severe phenotype. In fact, this patient shows a phenotype more severe than average, with a classical age-at-onset (10 years), but presenting an unusually fast progression to wheelchair by the age of 18 ; this may be due to the presence/aberrant trafficking of the mutant protein.

The large duplicated region found in patient 10 (at least from $B 2 M$ to CASC4, i.e., $300 \mathrm{~kb}$ ) does not seem to overly contribute to the patient's phenotype. Here, the four-exon deletion (31-34), in combination with the $2 \mathrm{bp}$ point mutation, appears to be responsible for the disease, resulting in the clinical signs compatible with an SPG11 phenotype. It appears that this complex rearrangement has arisen from an SPG11 allele missing exons 31-34, which was afterwards duplicated, encompassing a considerably large flanking region as MLPA has allowed us to determine.

There are several disorders associated with the 15q locus involving copy number variations that can comprise deletions, duplications, and even triplications-Angelman/Prader-Willi syndrome, 15q duplication syndrome, and 15q triplication ${ }^{32}-$ which altogether illustrate the high degree of instability in this chromosomal region.

\section{Alu-mediated SPG11 rearrangements}

Regarding the single or multiple exon deletions found in our set of patients, the identification of the same microhomology domains in other Alu elements spread throughout the SPG11 gene (Figure 3) gives rise to the hypothesis that different, yet similar, large-scale rearrangements may occur through recombination mediated by the various Alu elements.

Although the microhomology sequence involved in the deletion of exons 31-34 does not appear frequently in other Alu elements, as it happens in other cases, the same deletion has been found so far in five additional families of German and Dutch descent. ${ }^{4,10,22}$ This could suggest that the genomic architecture of this particular gene region is prone to the occurrence of this specific recombination, as we also found it in Portuguese patients.

Besides the deletions reported here, another has been mapped involving the loss of exons 37 to 39 in an Italian patient. ${ }^{11}$ Our analysis shows that this large gene rearrangement also overlaps with Alu elements and its breakpoints involve a microhomology sequence that is also present in five other Alu elements (Figure 2d).

For all SPG11 rearrangements mapped to date, the microhomology region adjacent to both breakpoints is located in the same relative position, closer to one or the other of the Alu extremities. The recombination event excises complementary parts of the Alu elements so that an Alu is left in the recombination spot and another leaves with the deleted portion; thus, these exchange genetic material with each other, allowing for an inter-/intrachromosomal form of nonallelic homologous recombination to take place (Figure 2). Our findings indicate that, although each deletion appears to be unique, Alu sequences are determinant for the instability observed at the SPG11 locus. The high frequency of Alu elements in SPG11 suggests a locus prone to de novo rearrangements; however, among six patients carrying these large deletions, parental segregation excluded a de novo event in five of the families. Notwithstanding, no information is available regarding the presence of $S P G 11$ rearrangements in the general healthy population.

\section{Evolutionary conservation of Alus involved in human SPG11 deletions in nonhuman primates}

To look at these Alu elements in the SPG11 gene from an evolutionary point of view, which could help to explain the genetic rearrangements we found, the SPG11 gene sequence of four nonhuman primates was analyzed (Figure 3 ). Our results show that the Alu elements containing the microhomology regions that are involved in the large deletions found in SPG11 patients are also present in nonhuman primates and conserved across evolution. These repetitive elements appear to have increased in number, at the SPG11 locus, throughout evolution, with the exception of the Gorilla, though this may be due to the gene not being fully sequenced in this species. Apparent to the observer is also the increase in elements belonging to the newest $A l u$ family, AluY ${ }^{33}$ Altogether, the increasing number of Alus present in the gene ultimately favors recombinatory events that might lead to a loss of segments of the SPG11 gene through an intra-/interchromosomal mechanism of nonallelic homologous recombination.

Transposable elements are highly frequent in the human genome, constituting up to $40 \%$ of its sequence. ${ }^{34}$ The majority of these elements lost their transposition ability a long time ago, although some of them, in particular, LINE-1 and Alu, have been shown to maintain this capacity, thus contributing to recent evolutionary history of the primate genomes ${ }^{35}$ and also to the genomic instability found to be involved in cancer and several genetic disorders. ${ }^{36}$

Similar to what happens with the BRCA1 gene, often known to suffer Alu-mediated deletions, ${ }^{37,38}$ analysis of SPG11 also shows a high density of interspersed repeats, namely Alu elements, which together with the large size of this gene $(\sim 101 \mathrm{~kb})$ favors the occurrence of various rearrangements. In addition, and similar to what seems to be happening with the SPG11 deletions, the Alu-mediated BRCA1 rearrangements arise from nonallelic homologous recombination. 


\section{Recombination hotspots are apparently unrelated to spatacsin rearrangements}

Recently, a degenerate 13-bp motif (CCNCCNTNNCCNC) was associated with recombination hotspots and genome instability in humans. ${ }^{39}$ Moreover, this motif was present in breakpoint regions of disease-causing nonallelic homologous recombination hotspots $^{40}$ and could, in theory, also be involved in SPG11 deletions. Through bioinformatic analysis, we found 13 degenerate sequences that occur 23 times over the gene's length, although none of them is located near any of the deletions identified in SPG11 patients. Nevertheless, other recombination events mediated by clusters of this motif might occur, more specifically in introns 18 and 20, where these elements seem to cluster.

\section{Conclusions}

In this study, we report 13 new families with SPG11, carrying both novel and previously reported mutations. We describe a complex entire SPG11 rearrangement and show that large gene rearrangements are frequent among patients with SPG11. In addition, we present the molecular characterization of three large SPG11 deletions together with their mechanisms and show a high frequency for $A l u$-mediated deletions in this gene. Our analysis suggests that the high number of repeated elements in SPG11 together with the presence of recombination hotspots and the high intrinsic instability of the $15 \mathrm{q}$ locus all contribute toward making this genomic region prone to large gene rearrangements.

Our findings enlarge the amount of data relating repeated masked elements to neurodegenerative disorders and highlight the importance of these elements in human disease and in evolution.

\section{ACKNOWLEDGMENTS}

We thank all patients and their families who participated in this study. We also thank all the physicians who referred HSP patients to our center. This work was supported by research grant PIC/IC/83232/2007, FCT (Fundação para a Ciência e Tecnologia), cofunded by FEDER (Fundo Europeu de Desenvolvimento Regional) and COMPETE (Programa Operacional Factores de Competitividade) and by Financiamento Plurianual de Unidades de Investigação (FCT). I.A. is funded by Programa Ciência, POPH - QREN - Tipologia 4.2 Promoção do Emprego Científico, cofunded by ESF, and national funds from the MCTES (Ministério da Ciência e Ensino Superior).

\section{DISCLOSURE}

The authors declared no conflict of interest.

\section{REFERENCES}

1. Boukhris A, Stevanin G, Feki I, et al. Hereditary spastic paraplegia with mental impairment and thin corpus callosum in Tunisia: SPG11, SPG15, and further genetic heterogeneity. Arch Neuro/ 2008;65:393-402.

2. Erichsen AK, Stevanin G, Denora P, Brice A, Tallaksen CM. SPG11-the most common type of recessive spastic paraplegia in Norway? Acta Neurol Scand, Supp/ 2008;188:46-50.

3. Stevanin G, Santorelli FM, Azzedine H, et al. Mutations in SPG11, encoding spatacsin, are a major cause of spastic paraplegia with thin corpus callosum. Nat Genet 2007;39:366-372.
4. Denora PS, Schlesinger D, Casali C, et al. Screening of ARHSP-TCC patients expands the spectrum of SPG11 mutations and includes a large scale gene deletion. Hum Mutat 2009;30:E500-E519.

5. Stevanin G, Azzedine H, Denora P, et al. Mutations in SPG11 are frequent in autosomal recessive spastic paraplegia with thin corpus callosum, cognitive decline and lower motor neuron degeneration. Brain 2008;131(Pt 3):772-784.

6. Murmu RP, Martin E, Rastetter A, et al. Cellular distribution and subcellular localization of spatacsin and spastizin, two proteins involved in hereditary spastic paraplegia. Mol Cell Neurosci 2011;47:191-202.

7. Slabicki $M$, Theis $M$, Krastev DB, et al. A genome-scale DNA repair RNA screen identifies SPG48 as a novel gene associated with hereditary spastic paraplegia. PLoS Bio/ 2010;8:e1000408.

8. Hanein S, Martin E, Boukhris A, et al. Identification of the SPG15 gene, encoding spastizin, as a frequent cause of complicated autosomalrecessive spastic paraplegia, including Kjellin syndrome. Am J Hum Genet 2008;82:992-1002.

9. Anheim M, Lagier-Tourenne C, Stevanin G, et al. SPG11 spastic paraplegia. A new cause of juvenile parkinsonism. J Neurol 2009;256:104-108.

10. Bauer $P$, Winner $B$, Schüle $R$, et al. Identification of a heterozygous genomic deletion in the spatacsin gene in SPG11 patients using high-resolution comparative genomic hybridization. Neurogenetics 2009;10:43-48.

11. Crimella C, Arnoldi A, Crippa F, et al. Point mutations and a large intragenic deletion in SPG11 in complicated spastic paraplegia without thin corpus callosum. J Med Genet 2009;46:345-351.

12. Del Bo R, Di Fonzo A, Ghezzi S, et al. SPG11: a consistent clinical phenotype in a family with homozygous spatacsin truncating mutation. Neurogenetics 2007:8:301-305

13. Hehr $U$, Bauer $P$, Winner $B$, et al. Long-term course and mutational spectrum of spatacsin-linked spastic paraplegia. Ann Neurol 2007;62:656-665.

14. Kim SM, Lee JS, Kim S, et al. Novel compound heterozygous mutations of the SPG11 gene in Korean families with hereditary spastic paraplegia with thin corpus callosum. J Neurol 2009;256:1714-1718.

15. Lee MJ, Cheng TW, Hua MS, et al. Mutations of the SPG11 gene in patients with autosomal recessive spastic paraparesis and thin corpus callosum. J Neurol Neurosurg Psychiatr 2008;79:607-609.

16. Liao SS, Shen L, Du J, et al. Novel mutations of the SPG11 gene in hereditary spastic paraplegia with thin corpus callosum. J Neurol Sci 2008;275: 92-99.

17. Orlén H, Melberg A, Raininko R, et al. SPG11 mutations cause Kjellin syndrome, a hereditary spastic paraplegia with thin corpus callosum and central retinal degeneration. Am J Med Genet B Neuropsychiatr Genet 2009;150B:984-992.

18. Paisan-Ruiz C, Dogu O, Yilmaz A, Houlden H, Singleton A. SPG1 1 mutations are common in familial cases of complicated hereditary spastic paraplegia. Neurology 2008;70(16 Pt 2):1384-1389.

19. Paisan-Ruiz C, Nath P, Wood NW, Singleton A, Houlden H. Clinical heterogeneity and genotype-phenotype correlations in hereditary spastic paraplegia because of spatacsin mutations (SPG11). Eur J Neurol 2008;15:1065-1070.

20. Pippucci T, Panza E, Pompilii E, et al. Autosomal recessive hereditary spastic paraplegia with thin corpus callosum: a novel mutation in the SPG11 gene and further evidence for genetic heterogeneity. Eur J Neurol 2009;16:121-126.

21. Samaranch L, Riverol M, Masdeu JC, et al. SPG11 compound mutations in spastic paraparesis with thin corpus callosum. Neurology 2008;71: 332-336.

22. Schüle R, Schlipf N, Synofzik M, et al. Frequency and phenotype of SPG11 and SPG15 in complicated hereditary spastic paraplegia. J Neurol Neurosurg Psychiatr 2009;80:1402-1404.

23. Southgate L, Dafou D, Hoyle J, et al. Novel SPG11 mutations in Asian kindreds and disruption of spatacsin function in the zebrafish. Neurogenetics 2010;11:379-389.

24. Miller SA, Dykes DD, Polesky HF. A simple salting out procedure for extracting DNA from human nucleated cells. Nucleic Acids Res 1988;16:1215.

25. Maccari G, Gemignani F, Landi S. COMPASSS (Complex Pattern of Sequence Search Software), a simple and effective tool for mining complex motifs in whole genomes. Bioinformatics 2010;26:1777-1778.

26. Thomas PD, Campbell MJ, Kejariwal A, et al. PANTHER: a library of protein families and subfamilies indexed by function. Genome Res 2003;13:2129-2141. 
27. Bromberg Y, Rost B. SNAP: predict effect of non-synonymous polymorphisms on function. Nucleic Acids Res 2007;35:3823-3835.

28. Ferrer-Costa C, Gelpí JL, Zamakola L, Parraga I, de la Cruz X, Orozco M. PMUT: a web-based tool for the annotation of pathological mutations on proteins. Bioinformatics 2005;21:3176-3178.

29. Capriotti E, Calabrese R, Casadio R. Predicting the insurgence of human genetic diseases associated to single point protein mutations with support vector machines and evolutionary information. Bioinformatics 2006;22:2729-2734

30. Holbrook JA, Neu-Yilik G, Hentze MW, Kulozik AE. Nonsense-mediated decay approaches the clinic. Nat Genet 2004;36:801-808.

31. Kang JQ, Shen W, Macdonald RL. Two molecular pathways (NMD and ERAD) contribute to a genetic epilepsy associated with the $G A B A(A)$ receptor GABRA1 PTC mutation, 975delC, S326fs328X. J Neurosci 2009;29:2833-2844.

32. Kumar D. Disorders of the genome architecture: a review. Genomic Med 2008:2:69-76.

33. Witherspoon DJ, Watkins WS, Zhang $Y$, et al. Alu repeats increase local recombination rates. BMC Genomics 2009;10:530.
34. Lander ES, Linton LM, Birren B, et al. Initial sequencing and analysis of the human genome. Nature 2001;409:860-921.

35. Deininger PL, Batzer MA. Mammalian retroelements. Genome Res 2002;12:1455-1465.

36. Cordaux R, Batzer MA. The impact of retrotransposons on human genome evolution. Nat Rev Genet 2009;10:691-703.

37. Mazoyer S. Genomic rearrangements in the BRCA1 and BRCA2 genes. Hum Mutat 2005;25:415-422.

38. Ticha I, Kleibl Z, Stribrna J, et al. Screening for genomic rearrangements in BRCA1 and BRCA2 genes in Czech high-risk breast/ovarian cancer patients: high proportion of population specific alterations in BRCA1 gene. Breast Cancer Res Treat 2010;124:337-347.

39. Myers S, Freeman C, Auton A, Donnelly P, McVean G. A common sequence motif associated with recombination hot spots and genome instability in humans. Nat Genet 2008;40:1124-1129.

40. Roehl AC, Vogt J, Mussotter T, et al. Intrachromosomal mitotic nonallelic homologous recombination is the major molecular mechanism underlying type-2 NF1 deletions. Hum Mutat 2010;31:1163-1173. 